\title{
San-Huang-Xie-Xin-Tang Prevents Rat Hearts from Ischemia/Reperfusion-Induced Apoptosis through eNOS and MAPK Pathways
}

\author{
Shu-Fen Liou, ${ }^{1}$ Hung-Jen Ke, ${ }^{2}$ Jong-Hau Hsu, ${ }^{3,4}$ Jyh-Chong Liang, ${ }^{5}$ Hung-Hong Lin, ${ }^{1}$ \\ Ing-Jun Chen, ${ }^{2}$ and Jwu-Lai Yeh ${ }^{2}$ \\ ${ }^{1}$ Department of Pharmacy, Chia-Nan University of Pharmacy and Science, Tainan, Taiwan \\ ${ }^{2}$ Department and Graduate Institute of Pharmacology, Kaohsiung Medical University, Kaohsiung 807, Taiwan \\ ${ }^{3}$ Department of Paediatrics, Faculty of Medicine, College of Medicine, Kaohsiung Medical University, Taiwan \\ ${ }^{4}$ Department of Paediatrics, Kaohsiung Medical University Hospital, Kaohsiung, Taiwan \\ ${ }^{5}$ Graduate Institute of Engineering, National Taiwan University of Science and Technology, Taipei, Taiwan \\ Correspondence should be addressed to Jwu-Lai Yeh, jwulai@kmu.edu.tw
}

Received 1 December 2009; Accepted 20 April 2010

Copyright () 2011 Shu-Fen Liou et al. This is an open access article distributed under the Creative Commons Attribution License, which permits unrestricted use, distribution, and reproduction in any medium, provided the original work is properly cited.

San-Huang-Xie-Xin-Tang (SHXT) is a traditional Chinese medication consisting of three herbs, namely Coptidis rhizome, Scutellariae radix and Rhei rhizome. This study aimed to examine the cardioprotective effects of SHXT in a rat model of acute myocardial apoptosis induced by ischemia/reperfusion (I/R). Vehicle (intravenous saline) or SHXT (intravenous or oral) was administered prior to I/R (occlusion of left coronary artery for $45 \mathrm{~min}$ followed by reperfusion for $2 \mathrm{~h}$ ). In the vehicle group, myocardial I/R caused myocardial infarction with increased plasma cardiac enzymes, severe arrhythmia and mortality. Myocardial apoptosis was induced by I/R as evidenced by DNA ladder and Bcl-2/Bax ratio. In the SHXT group, we found that SHXT significantly reduced plasma levels of cardiac enzymes, arrhythmia scores (from $5 \pm 1$ to $2 \pm 1, P<.01$ ) and mortality rate (from 53 to $0 \%, P<.01$ ). In addition, pretreatment with intravenous SHXT reduced the infarct size dose-dependently when compared with the vehicle group $\left(10 \mathrm{mg} \mathrm{kg}^{-1}: 14.0 \pm 0.2\right.$ versus $44.5 \pm 5.0 \%$, and $30 \mathrm{mg} \mathrm{kg}^{-1}: 6.2 \pm 1.2 \%$ versus $44.5 \pm 5.0 \%$, both $P<.01)$. Similarly, oral administration of SHXT reduced the infarct size dose-dependently. Furthermore, SHXT markedly decreased the apoptosis induced by I/R with increased Bcl-2/Bax ratio. Finally, we found that SHXT counteracted the I/R-induced downstream signaling, resulting in increased myocardial eNOS expression and plasma nitrite, and decreased activation of ERK1/2, p38 and JNK. These data suggest that SHXT has cardioprotective effects against I/R-induced apoptosis, and that these effects are mediated, at least in part, by eNOS and MAPK pathways.

\section{Introduction}

Myocardial injury from ischemia/reperfusion (I/R) is a clinical problem requiring invasive interventions such as thrombolysis, angioplasty and coronary bypass surgery, to reestablish coronary blood flow and minimize cardiac damage due to severe myocardial ischemia [1]. Reperfusion of an occluded coronary artery is known to reduce infarct size, preserve left ventricular function, and reduce overall mortality. However, it is now recognized that the readmission of oxygenated blood into previously ischemic myocardium can initiate a cascade of events that will paradoxically produce additional myocardial cell dysfunction and cell apoptosis [1-3].
San-Huang-Xie-Xin-Tang (SHXT), a widely used traditional Chinese medication, consists of three herbs, namely Coptidis rhizome (Coptis chinesis Franch), Scutellariae radix (Scutellaria baicalensis Georgi) and Rhei rhizome (Rheum officinale Baill). Previous laboratory and clinical studies have suggested that SHXT may have a role in the treatment of various diseases including gastrointestinal disorders [4], acute lung injury [5], septic shock [6], hypertension $[7,8]$ and neuronal injury [9]. There are three major bioactive constituents in SHXT: berberine, baicalin and baicalein [10, 11]. Berberine, one of the main components in Coptidis rhizoma, has been shown to improve cardiac performance and decrease ventricular premature complexes and mortality in 
patients with congestive heart failure $[12,13]$. In addition, it was recently found that berberine can reduce I/R injury and attenuate I/R-induced apoptosis in myocytes [14]. Baicalin and baicalein are both flavonoids derived from the root of Scutellaria baicalensis, and have been reported to protect rat cardiomyocytes against hypoxia/reoxygenation and I/R, partly due to their scavenging capacity for reactive oxygen species (ROS) generated during these insults $[15,16]$. Furthermore, Rhei rhizoma extracts reportedly possess antioxidant and anti-lipid peroxidation activities through their direct suppression of mitochondrial ROS generation [17].

Even though the individual effects of these three major bioactive constituents of SHXT have been found to be beneficial in the settings of congestive heart failure or I/R-induced myocardial damage [12-16], no studies have examined whether the whole compound of SHXT also conveys cardioprotective effects. Thus, the present study aimed to investigate whether pretreatment with SHXT protects rat hearts against I/R-induced myocardial apoptosis, and if so, whether the anti-apoptotic effects are mediated by nitric oxide (NO) and mitogen-activation protein kinase (MAPK) pathways.

\section{Methods}

2.1. Materials and Reagents. The voucher specimens and methods of extraction and analysis of SHXT were the same as we have previously described [5]. Reagents used in this study included: antibodies of eNOS and $\beta$-actin from SigmaAldrich Inc. (St. Louis, MO, USA); antibodies of Bcl-2, Bax, ERK1/2, phosphorylated ERK1/2, phosphorylated p38, JNK and phosphorylated JNK from Upstate Biotechnology (Lake Placid, NY, USA); antibody of p38 from Santa Cruz Biotech (Santa Cruz, CA, USA); PVDF membrane and an enhanced chemiluminescence (ECL) kit from PerkinElmer Life and Analytical Sciences (Waltham, MA, USA), and peroxidaseconjugated immunoglobulins from Calbiochem (Temecula, CA, USA).

2.2. Experimental Animals. Wistar rats from the National Laboratory Animal Breeding and Research Center (Taipei, Taiwan) were housed under conditions of constant temperature and controlled illumination (light on between 7:30 and 19:30 hours). Food and water were available ad libitum. The study was approved by the Animal Care and Use Committee of the Kaohsiung Medical University.

2.3. Experimental Protocol. The I/R protocol was performed as we have previously described [18]. In brief, male Wistar rats weighing 250-300 g were anesthetized with intraperitoneal pentobarbital sodium $\left(40 \mathrm{mg} \mathrm{kg}^{-1}\right)$. Tracheotomy was performed and an intubating cannula was connected to a rodent ventilator. The animals were ventilated artificially with room air. The left femoral artery and vein were cannulated for the measurement of arterial blood pressure $(\mathrm{ABP})$ and heart rate $(\mathrm{HR})$ via a Statham pressure transducer and a Biotechnometer (AD Instruments, Mountain View, CA, USA), and for the administration of drugs, respectively. After a left-side thoracotomy was performed at the fourth intercostal space, the pericardium was incised and the heart was exteriorized. A ligature (6/0 silk suture) was placed around the left main coronary artery close to its origin. The thread was then made into a knot as an occluder and another thread was tied to the first knot as a releaser. The ends of both threads were brought outside the thoracic cavity. Thus, the occlusion could be tightened or loosened by pulling the thread of the releaser. The coronary artery was occluded for $45 \mathrm{~min}$, followed by $120 \mathrm{~min}$ of reperfusion. Sham-operated animals (sham group) underwent all of the above-described surgical procedures except that the $6 / 0$ silk was passed around the left coronary artery, but was not tied.

SHXT was prepared according to the method we have described previously [5]. Animals were randomized into the following groups: (i) sham, (ii) operated + vehicle (i.v. saline), (iii) operated + SHXT (10 $\mathrm{mg} \mathrm{kg}^{-1}$, i.v. bolus; $15 \mathrm{~min}$ prior to ischemia), (iv) SHXT (30 $\mathrm{mg} \mathrm{kg}^{-1}$, i.v. bolus), (v) SHXT (10 mg kg-1, oral; $1 \mathrm{~h}$ prior to ischemia), (vi) SHXT (30 $\mathrm{mg} \mathrm{kg}^{-1}$, oral).

2.4. Evaluation of Arrhythmia and Mortality. For all the groups, heart rate was measured from the recordings of an electrocardiogram (lead II) and the incidence of arrhythmia registered in accordance with the Lambeth Conventions [19] as ventricular tachycardia (VT), ventricular fibrillation (VF) and ventricular ectopic beat (VEB). VEB is defined as a discrete and identifiable premature QRS complex. VT was diagnosed as four or more consecutive VEBs. VF was diagnosed when the ECG recording showed chaotic activity with an amplitude less than that of the normal ECG. Complex forms (e.g. bigeminy) were included in the count of VEB and not analyzed separately. VF may be sustained or may revert spontaneously to a normal sinus rhythm in rats. Irreversible VF was defined as VF which did not reverse within $5 \mathrm{~min}$ of onset. The onset and duration of arrhythmia were also measured. The arrhythmia score for these experiments was calculated using a previously published scale [20]. The mortality rate was also recorded in each group.

2.5. Determination of Myocardial Infarct Size. At the end of the experiment the coronary artery was occluded and $0.5 \mathrm{~mL}$ of 5\% Evans blue was injected intravenously to visualize the area at risk of infarction (AAR). The heart was then excised and the atria were removed. The entire ventricular area was sectioned into 2- to 3-mm thick slices from the apex to the base and incubated in 1\% triphenyltetrazolium chloride (TTC) for $15 \mathrm{~min}$ at $37^{\circ} \mathrm{C}$. TTC stained the viable tissue dark red while the infarcted tissue remained grayish-white. The AAR and area of infarct were both traced by hand on an imaging system. The traced areas were then quantified by computerized planimetry [18].

2.6. Measurement of Plasma LDH, CK-MB and Troponin I. Myocardial cellular damage and necrosis were evaluated by measuring plasma levels of three cardiac enzymes: lactate dehydrogenase (LDH), troponin I and creatine kinase (CK)$\mathrm{MB}$. The blood samples were drawn from the femoral artery at the end of reperfusion and collected in heparinized tubes. 
TABLE 1: Effect of SHXT on the severity of arrhythmias induced by ischemia/reperfusion in anesthetized rats.

\begin{tabular}{|c|c|c|c|c|c|c|c|c|}
\hline Groups $(n=8)$ & Total VEBs & $\begin{array}{c}\text { Duration of } \\
\text { VT (s) }\end{array}$ & $\begin{array}{c}\text { Duration of } \\
\text { VF (s) }\end{array}$ & $\mathrm{VT}(\%)$ & VF (\%) & $\begin{array}{c}\text { Irreversible } \\
\text { VF (\%) }\end{array}$ & $\begin{array}{c}\text { Mortality } \\
(\%)\end{array}$ & Scores \\
\hline Sham & $63 \pm 6$ & 0 & 0 & 0 & 0 & 0 & 0 & $1 \pm 1$ \\
\hline $\mathrm{I} / \mathrm{R}+$ vehicle & $781 \pm 49^{* *}$ & $67 \pm 3^{* *}$ & $23 \pm 3^{* *}$ & 100 & 83 & 17 & 53 & $5 \pm 1^{* *}$ \\
\hline $\mathrm{I} / \mathrm{R}+\mathrm{SHXT}(10 \mathrm{mg} / \mathrm{kg}$, i.v. $)$ & $326 \pm 54^{\ddagger}$ & $37 \pm 8^{+}$ & $10 \pm 3^{+}$ & 100 & 33 & 0 & 0 & $3 \pm 1^{\ddagger}$ \\
\hline $\mathrm{I} / \mathrm{R}+\operatorname{SHXT}(30 \mathrm{mg} / \mathrm{kg}$, i.v. $)$ & $166 \pm 28^{\ddagger}$ & $19 \pm 3^{\ddagger}$ & $3 \pm 1^{\ddagger}$ & 67 & 33 & 0 & 0 & $2 \pm 1^{\ddagger}$ \\
\hline $\mathrm{I} / \mathrm{R}+\mathrm{SHXT}(10 \mathrm{mg} / \mathrm{kg}$, oral $)$ & $535 \pm 35^{+}$ & $42 \pm 4^{+}$ & $20 \pm 5$ & 100 & 83 & 0 & 33 & $4 \pm 1$ \\
\hline $\mathrm{I} / \mathrm{R}+\mathrm{SHXT}(30 \mathrm{mg} / \mathrm{kg}$, oral $)$ & $210 \pm 30^{\ddagger}$ & $21 \pm 3^{\ddagger}$ & $6 \pm 1^{\ddagger}$ & 67 & 33 & 0 & 0 & $2 \pm 1^{\ddagger}$ \\
\hline
\end{tabular}

Values are mean \pm SEM. Evaluation of arrhythmias described in Methods section. VEB: ventricular ectopic beats; VT: ventricular tachycardia; VF: ventricular fibrillation; I/R: ischemia/reperfusion; SHXT: San-Huang-Xie-Xin-Tang. ANOVA followed by Dunnett's test.

${ }^{* *} P<.01$ versus sham group; ${ }^{+} P<.05,{ }^{\ddagger} P<.01$ versus vehicle-treated rats.

Samples were promptly centrifuged at $2000 \mathrm{~g}$ for $15 \mathrm{~min}$ until measurements. Plasma levels of LDH and CK-MB were measured with a commercial kit from Sigma-Aldrich Inc. Serum troponin I was measured with a commercial third-generation electrochemiluminescence immunoassay kit (Roche Diagnostics, Indianapolis, IN, USA).

2.7. Determination of Plasma Nitrite Production. Blood samples were collected at the end of the experiment. Samples were promptly centrifuged at $2000 \mathrm{~g}$ for $20 \mathrm{~min}$ at $4^{\circ} \mathrm{C}$. Plasma supernatant was stored at $-80^{\circ} \mathrm{C}$ until analyzed. $\mathrm{NO}$ production was spectrophotometrically determined by assaying the plasma supernatant for nitrite using the Griess reagent $(1 \%$ sulfanilamide and $0.1 \%$ naphthylethylenediamide in $5 \%$ phosphoric acid). Absorbance was measured at $540 \mathrm{~nm}$ and nitrite concentration was determined using sodium nitrite as a standard.

2.8. Detection of DNA Fragmentation. Myocardial apoptosis was qualitatively analyzed by detection of DNA fragmentation. Genomic DNA from fresh-frozen tissue was isolated using a Purgene DNA Isolation Kit (Purgene, Minneapolis, $\mathrm{MN}$, USA) according to the manufacturer's instructions. Twenty-five micrograms of DNA was loaded onto $2 \%$ agarose gel containing $0.5 \mu \mathrm{g} \mathrm{mL} \mathrm{m}^{-1}$ ethidium bromide. DNA electrophoresis was carried out at $50 \mathrm{~V}$ for 1-2 h. DNA ladder formation, a hallmark of tissue apoptosis, was visualized under ultraviolet light and photographed for a permanent record.

2.9. Western Blotting. Left ventricles were removed at the end of the experiment, and lysed with lysis buffer $[20 \mathrm{mM}$ Tris- $\mathrm{HCl}$ (pH 7.5), $1 \mathrm{mM}$ dithiothreitol (DTT), 5 mM EGTA, $2 \mathrm{mM}$ EDTA, $0.5 \mathrm{mM}$ PMSF, $20 \mu \mathrm{M}$ leupeptin and $20 \mu \mathrm{M}$ aprotinin]. After sonication, the lysates were centrifuged and the proteins were separated by electrophoresis on SDS-PAGE $(10-14 \%)$ and transferred onto a polyvinylidene difluoride (PVDF)-plus membrane. After blocking for $1 \mathrm{~h}$ in $5 \%$ nonfat dry milk in Tris-buffered saline, the membrane was incubated with the desired primary antibody for $2 \mathrm{~h}$. The membrane was then treated with appropriate horseradish peroxidase conjugated secondary antibody (diluted $1: 1000$ ), and the immunoreactive bands were detected with enhanced chemiluminescence reagents (PerkinElmer Life and Analytical Sciences).

2.10. Statistical Analysis. The results are expressed as mean \pm SEM. Statistical differences were estimated by one-way analysis of variance (ANOVA) followed by Dunnett's test. A value of $P<.05$ was considered significant.

\section{Results}

3.1. Ventricular Arrhythmia and Mortality Rate. SHXT produced markedly antiarrhythmic effects in anesthetized rats as shown in Table 1. I/R caused pronounced arrhythmogenic activity with $100 \%$ VT and 63\% VF. In both oral $\left(10 \mathrm{mg} \mathrm{kg}^{-1}\right)$ and i.v. bolus SHXT $\left(10 \mathrm{mg} \mathrm{kg}^{-1}\right)$ treated groups, there were significant decreases in the number of VEBs (from $781 \pm 49$ to $535 \pm 35$ and $326 \pm 54$, resp., $P<.05$ ) and the incidence of VF (both from 63 to 25\%) and irreversible VF (both from 13 to $0 \%$ ). Similarly, both oral $\left(30 \mathrm{mg} \mathrm{kg}^{-1}\right)$ and i.v. bolus administration of SHXT $\left(30 \mathrm{mg} \mathrm{kg}^{-1}\right.$ ) significantly reduced the total number of VEBs (from $781 \pm 49$ to $210 \pm 30$ and $166 \pm 28$, resp., $P<.01$ ) and the incidence of VT (both from 100 to $75 \%$ ), VF (both from 63 to 25\%) and irreversible VF (both from 13 to $0 \%$ ). Moreover, the duration of VT and VF was also significantly reduced in all SHXT-treated groups except for the group with low dose oral administration $\left(10 \mathrm{mg} \mathrm{kg}^{-1}\right)$. The most marked reductions in the arrhythmia scores were observed in high dose $\left(30 \mathrm{mg} \mathrm{kg}^{-1}\right)$ groups with oral and i.v. bolus administration (both from $5 \pm 1$ to $2 \pm 1, P<.01$ ). More importantly, SHXT decreased the mortality rate from 53 to $0 \%(P<.01)$.

3.2. Myocardial Infarct Size. SHXT has protective effects against myocardial infarction, as shown in Figure 1(a). There were no significant differences in the size of AAR among all groups. The vehicle group had a high proportion of infarcted tissue (INF/AAR ratio) of $44.5 \pm 5.0 \%$, which was significantly reduced by pretreatment with SHXT. Intravenous administration of SHXT at doses of 10 and $30 \mathrm{mg} \mathrm{kg}^{-1}$ resulted in INF/AAR ratios of $14.0 \pm 0.2 \%(P<.01)$ and $6.2 \pm 1.2 \%(P<.01)$, respectively. Oral administration of 


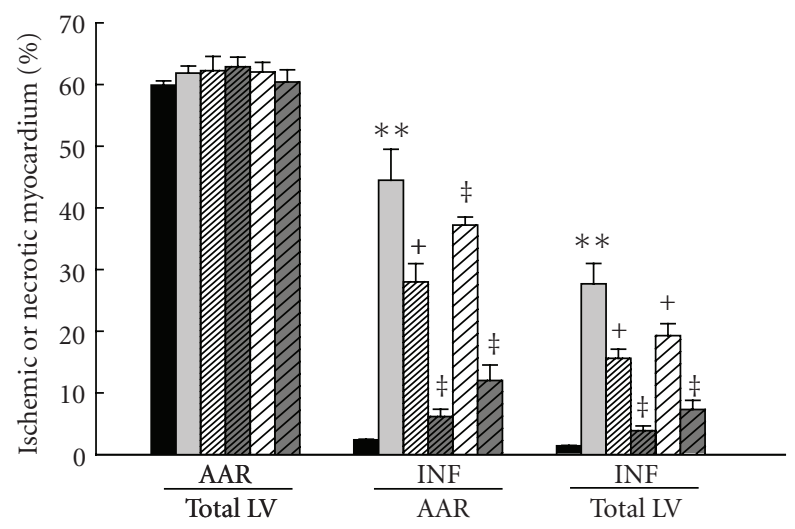

(a)
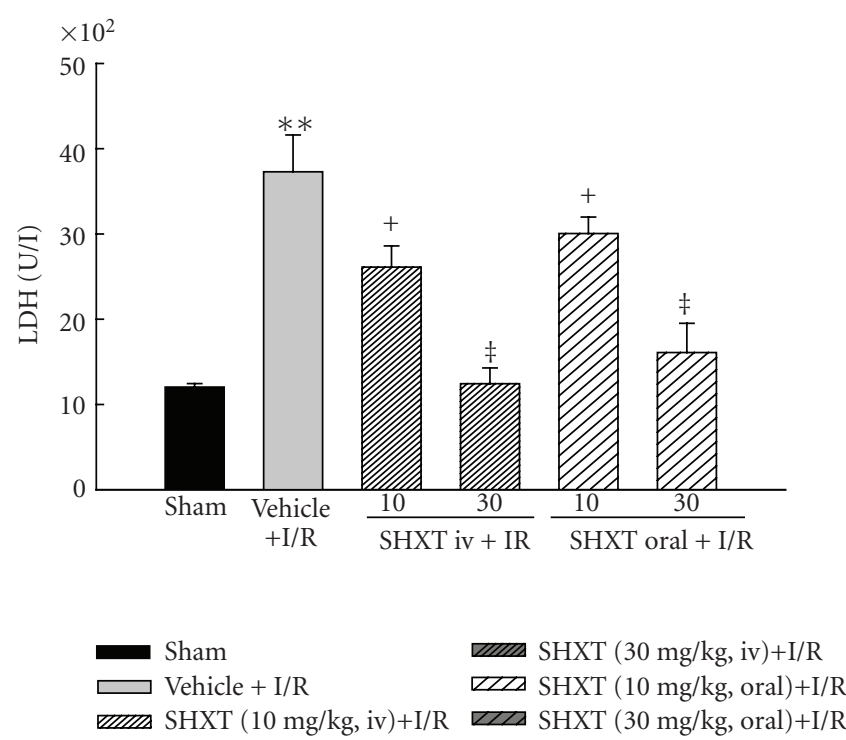

(c)

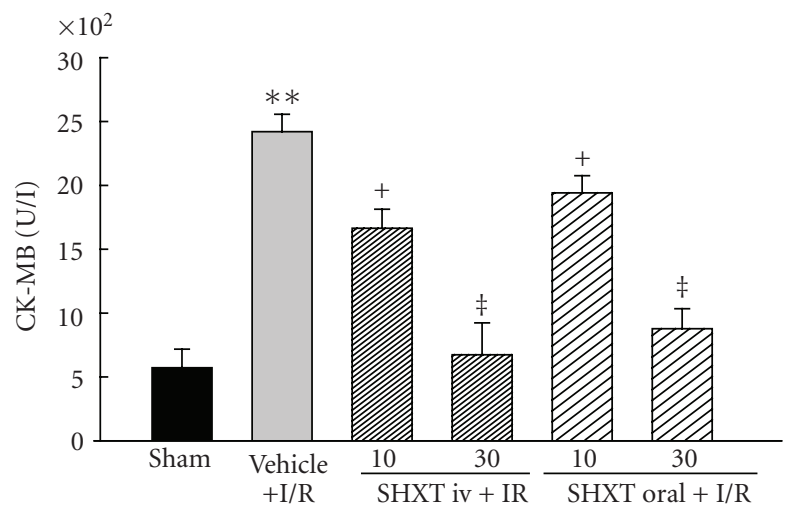

(b)
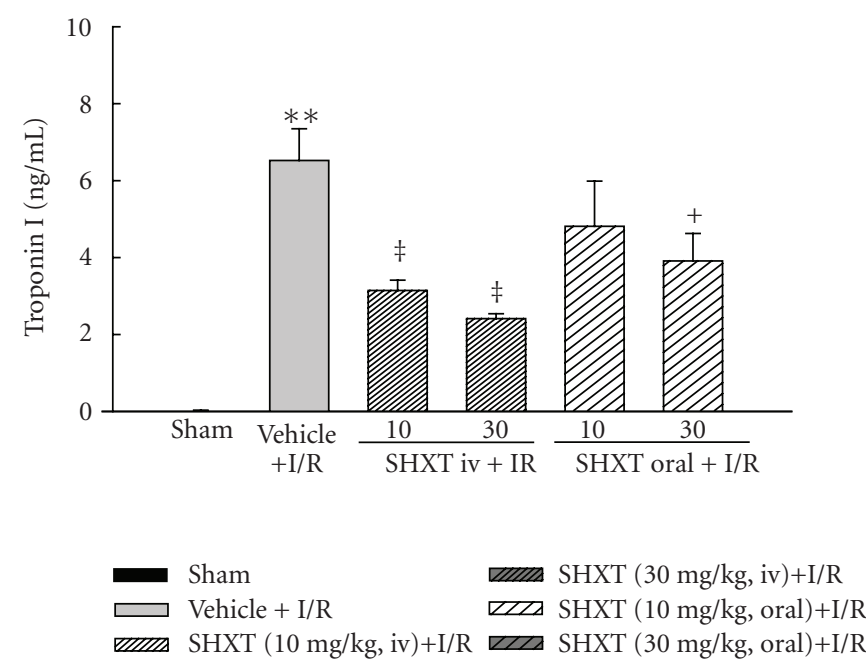

(d)

FIGURE 1: Effects of SHXT on myocardial infarct size and plasma levels of CK-MB, LDH and troponin I in rat hearts subjected to I/R. (a) Bar graphs show area at risk of infarction indexed to total left ventricle (AAR/Total LV), infarcted area indexed to area at risk of (INF/AAR), and infarcted area indexed to total left ventricle (INF/Total LV). (b)-(d) Effects of pretreatment with SHXT on plasma levels of cardiac enzymes. Each value represents the mean \pm SEM of six rats. ${ }^{* *} P<.01$ versus sham group; ${ }^{+} P<.05,{ }^{\ddagger} P<.01$ versus vehicle-treated rats. ANOVA followed by Dunnett's test.

SHXT also dose-dependently reduced this ratio. Similarly, the INF/total LV ratio was also significantly lower in rats with SHXT-treatment than in vehicle-treated rats.

3.3. Plasma $C K-M B, L D H$ and Troponin I. As shown in Figures $1(\mathrm{~b})-1(\mathrm{~d})$, in the sham group, all three cardiac enzymes remained at low levels at the end of surgery, indicating that a sham-operation without I/R did not result in significant myocardial injury. In the vehicle group, I/R caused increased levels of all these cardiac enzymes. However, pretreatment with SHXT by intravenous injection and oral administration both attenuated the increases of all these cardiac enzymes, in a concentration-dependent manner.

3.4. Myocardial DNA Fragmentation. In the sham group, there was no DNA fragmentation detected in myocardial tissue (Figure 2(a), lane 1). In contrast, the formation of DNA nucleosome fragmentation was clearly detected in myocardial tissues in the vehicle group (Figure 2(a), lane 2 ). Both intravenous and oral administration of high dose (30 $\mathrm{mg} \mathrm{kg}^{-1}$ ) SHXT markedly decreased myocardial DNA fragmentation (Figure 2(a), lanes 4 and 6).

3.5. Myocardial Expression of Bcl-2 and Bax. As shown in Figure 2(b), I/R caused decreased expression of Bcl-2 protein and increased expression of Bax protein in myocardium, resulting in a decrease of the $\mathrm{Bcl}-2 / \mathrm{Bax}$ ratio, indicating I/R-induced apoptosis. However, intravenous administration of SHXT prevented the decrease of the $\mathrm{Bcl}-2 / \mathrm{Bax}$ ratio dose-dependently. Similarly, but to a lesser extent, oral administration of SHXT at a high dose attenuated the decrease of the $\mathrm{Bcl}-2 / \mathrm{Bax}$ ratio. 


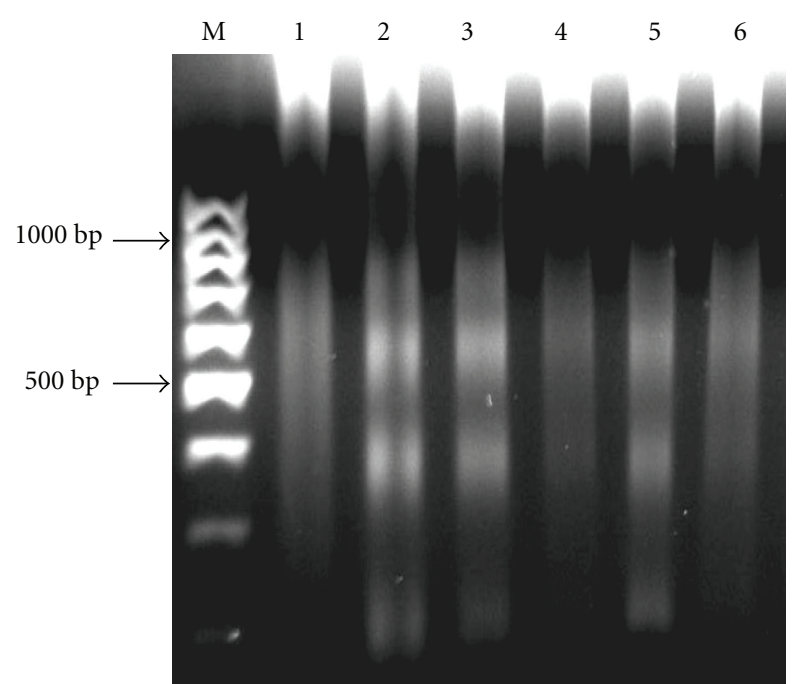

(a)
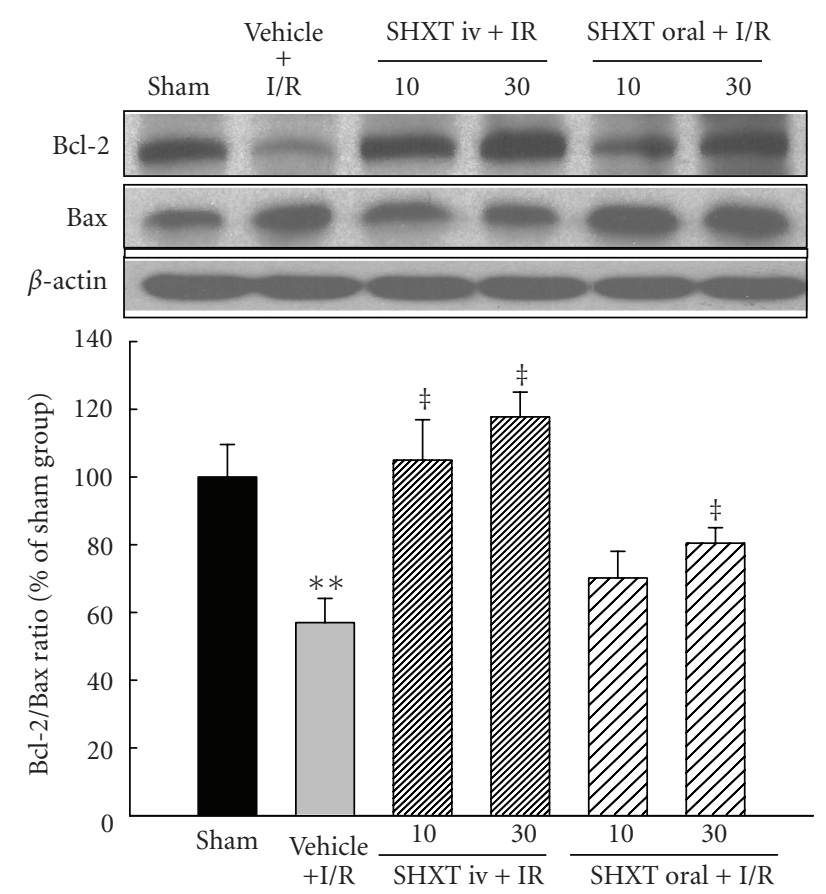

(b)

Figure 2: SHXT attenuated ischemia/reperfusion (I/R)-induced myocardial apoptosis by the DNA ladder and Bcl-2/Bax ratio. (a) DNA fragmentation was detected by agarose gel electrophoresis. $\mathrm{M}$ represents marker; lane 1 represents sham tissue; lane 2 represents vehicle-treated tissue; lane 3 represents SHXT $10 \mathrm{mg} / \mathrm{kg}$-treated tissue (i.v.); lane 4 represents SHXT $30 \mathrm{mg} / \mathrm{kg}$-treated tissue (i.v.); lane 5 represents SHXT $10 \mathrm{mg} / \mathrm{kg}$-treated tissue (oral); lane 6 represents SHXT $30 \mathrm{mg} / \mathrm{kg}$-treated tissue (oral). Similar results were obtained in four other experiments. (b) Quantitative analysis on the $\mathrm{Bcl}-2 / \mathrm{Bax}$ ratio by western blots. Each value represents the mean \pm SEM of six rats. ${ }^{* *} P<.01$ versus sham group; ${ }^{+} P<.05$, ${ }^{\ddagger} P<.01$ versus vehicle-treated rats. ANOVA followed by Dunnett's test.
3.6. Myocardial eNOS Protein and Plasma NO Production. As shown in Figure 3(a), I/R decreased the expression of myocardial eNOS protein. However, both intravenous injection and oral administration of SHXT prevented the decrease dose-dependently. Similarly, as shown in Figure 3(b), I/R caused a decreased level of plasma nitrite, and SHXT had a significantly antagonizing effect on this change, in a dosedependent manner.

3.7. MAPK Pathway. We finally explored the signaling pathway of SHXT-mediated anti-apoptotic effects, and examined the MAPK cascades, the upstream signaling molecules in apoptotic reactions. As shown in Figure 4(a), in comparison with the sham group, the phosphorylation of ERK1/2 was increased in the vehicle group due to I/R. However, the I/Rinduced activation of ERK1/2 was significantly abolished by pretreatment with SHXT. In addition, SHXT also attenuated the activation of p38 induced by I/R (Figure 4(b)), even though this effect was not significant when a low dose (10 $\mathrm{mg} \mathrm{kg}^{-1}$ ) was administered orally. Similarly, I/R-induced activation of JNK was attenuated by SHXT (Figure 4(c)).

\section{Discussion}

This is the first study to demonstrate that SHXT, a traditional Chinese medicinal formula, has composite cardioprotective effects against I/R of antiarrhythmia, reducing infarct size and increased survival in rats. In addition, SHXT possesses a potent inhibitory effect against I/R-induced myocardial apoptosis, which may be mediated, at least in part, by eNOS and MAPK pathways.

In our rat model with myocardial damage induced by $\mathrm{I} / \mathrm{R}$, we first found that pretreatment with SHXT significantly decreased the rhythm disturbances, mortality rate and myocardial infarct size. During an ischemic insult, myocytes may release a variety of intracellular enzymes and proteins into the blood. In the present study, we examined multiple biochemical markers including $\mathrm{CK}-\mathrm{MB}, \mathrm{LDH}$ and troponin I to detect cardiac cellular damage caused by $\mathrm{I} / \mathrm{R}$. We found that I/R-induced elevations of plasma LDH, CK-MB and troponin I were markedly blunted when rats were pretreated with SHXT.

Two distinct types of myocardium cell death, necrosis and apoptosis, have been linked with I/R-induced myocardial injury. Internucleosomal DNA fragmentation observed in agarose gels [21], chromatin condensation observed by Hoechst 33342 staining and flow cytometric analysis for translocation of phospholipid phosphatidylserine by annexin V/PI staining [22], have been widely used to define myocardial apoptosis. The mitochondria apoptotic pathway has been described as important for apoptotic cell death signaling for mammalian cells [23]. Apoptosis after I/R has been found to be associated with increased levels of Bax protein and also with a decreased Bcl-2/Bax ratio [24]. The overexpression of $\mathrm{Bcl}-2$ in mice can significantly inhibit apoptosis and decrease infarct size in the heart after I/R [25]. Therefore, we investigated the role of $\mathrm{Bcl}-2$ family proteins in the anti-apoptotic effects of SHXT. Our results showed 

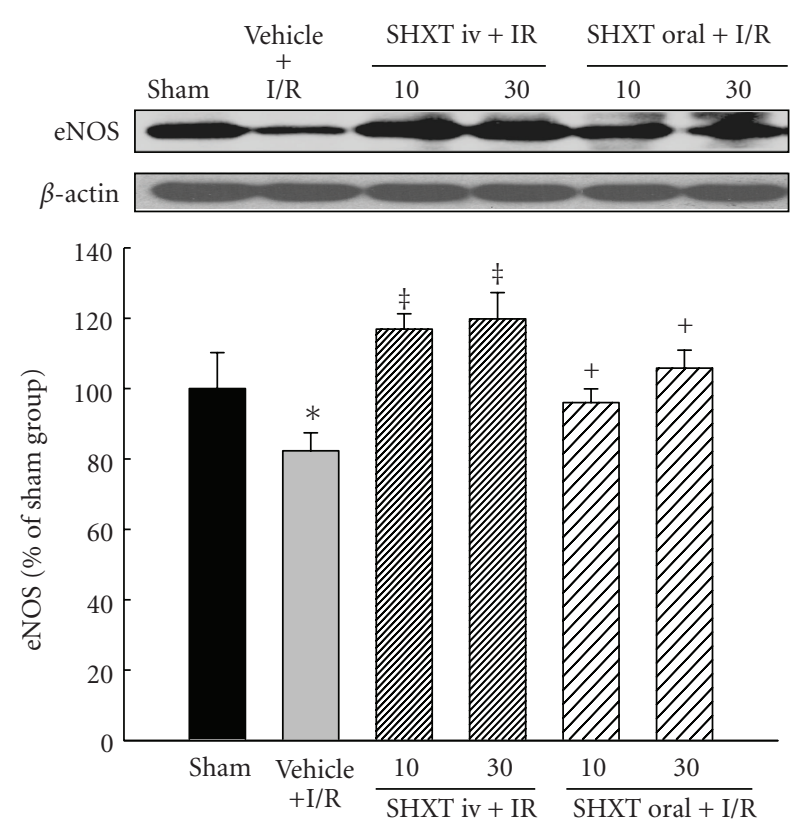

(a)

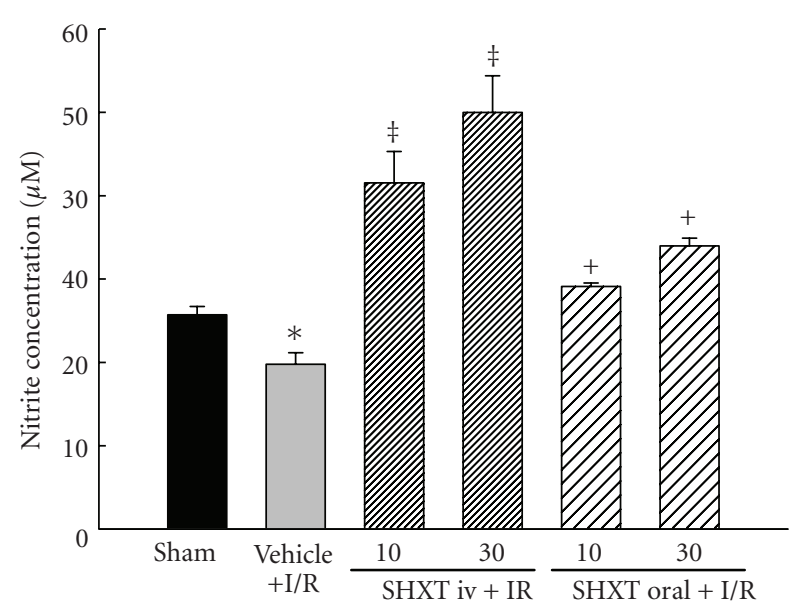

(b)

FIGURE 3: SHXT up-regulated eNOS protein expression and then promoted plasma NO production after I/R. (a) The expression level of eNOS protein in infarcted myocardial tissues was measured by immunoblot assay. The relative ratio of protein $/ \beta$-actin protein was quantified by densitometric analyses. (b) NO production was detected spectrophotometrically by measuring its metabolite, nitrite. Each value represents the mean \pm SEM of six rats. ${ }^{*} P<.05$ versus sham group; ${ }^{+} P<.05,{ }^{\ddagger} P<.01$ versus vehicle-treated rats. ANOVA followed by Dunnett's test.

that SHXT treatment significantly increased Bcl-2 expression and decreased Bax, suggesting that changes in the ratio of proapoptotic and antiapoptotic Bcl-2 family proteins may contribute to the antiapoptotic and cardioprotective effects of SHXT on I/R injury (Figure 5).

Numerous studies have demonstrated the cardioprotective effects of NO during I/R. In addition, it has been demonstrated that the effects of NO on myocardial
I/R injury include both pro- and anti-apoptotic effects depending on the source of NO $[26,27]$. However, precise mechanisms underlying these effects are complex and not completely understood. For example, in $\mathrm{eNOS}^{-/-}$mice, myocardial injury was found to be exacerbated following $\mathrm{I} / \mathrm{R}$, suggesting the protective role of eNOS-derived NO $[28,29]$. Additionally, treatment with statins has been found to reduce I/R injury after myocardial infarction, via PI3kinase/Akt and eNOS-mediated pathways with increased NO availability [30]. Some studies suggest that the beneficial effects of NO from eNOS are mediated by regulation of vascular tone, superoxide radical scavenging and inhibition of neutrophil adherence and infiltration [27-31]. Our results were consistent with previous studies showing that the myocardial expression of the eNOS protein was markedly decreased after I/R injury. In addition, we showed that administration of SHXT increased the expression of the eNOS protein, promoted NO generation and attenuated myocardial apoptosis in the I/R animal model. Collectively, the present study suggests that SXHT may protect myocardial tissue from apoptosis by modulating eNOS expression (Figure 5). Our results are in line with a recent study showing that hydroxysafflor yellow A, a flavonoid of Carthamus tinctorius extracts, can inhibit I/R-induced opening of mitochondrial permeability transition pores through enhanced NO production by eNOS activation [31].

Emerging evidence suggests that MAPK-signaling cascades play an important role in oxidative stress-induced apoptotic cell death $[31,32]$. The MAPK family has been classified into three major subfamilies: the extracellular signal-regulated kinase (ERK), the c-Jun N-terminal kinase (JNK) and the p38 MAPK. They are activated in response to myocardial $\mathrm{I} / \mathrm{R}$, and it is generally believed that the activations of ERK1/2 (beneficial) and p38 MAPKs-JNKs (deleterious) exert opposite effects on post-ischemic myocardial apoptosis and cardiac function recovery [33, 34]. Namely, activation of ERK contributes to cell differentiation, proliferation and survival, whereas JNK and p38 are activated by pro-inflammatory cytokines and environmental stresses and promote apoptosis [33]. However, there is some evidence suggesting that ERK1/2 also contributes to cell death in many organ systems. For example, ERK1/2 was activated in an animal model of I/R-induced brain injury, and inactivation of ERK1/2 reduced the extent of tissue damage $[35,36]$. In cardiomyocytes, a recent study has also shown activation of ERK $1 / 2$ to be important in Bcl-2 family-mediated cell apoptosis caused by doxorubicin [37]. Therefore, we finally examined the potential effects of SHXT on the activation of the different MAPK cascades induced by $\mathrm{I} / \mathrm{R}$. We found that activations of ERK1/2, p38 and JNK were observed in rat hearts subjected to $I / R$, and pretreatment with SHXT significantly decreased the activation of these activations. Our results indicate that SHXT suppresses activations of all three subfamilies of MAPK-signaling cascades induced by myocardial I/R injury. Even though these data suggest that SHXT may inhibit the survival action of ERK1/2, we speculate that this effect may be offset by p 38 and JNK, with the net effect of MAPKs potentially contributing to the antiapoptotic action of SHXT. 

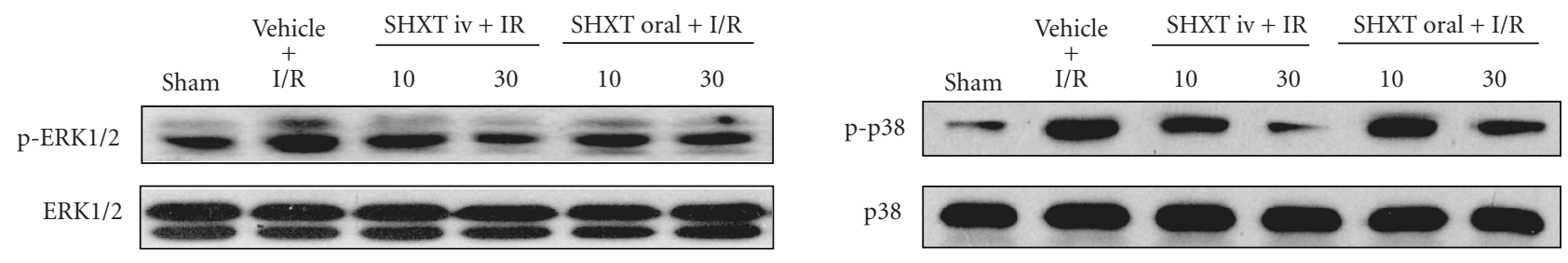

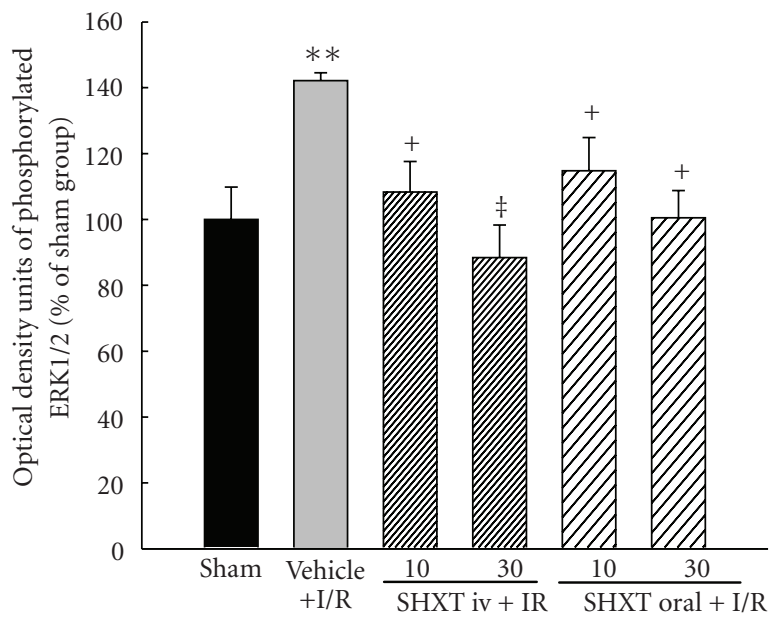

(a)

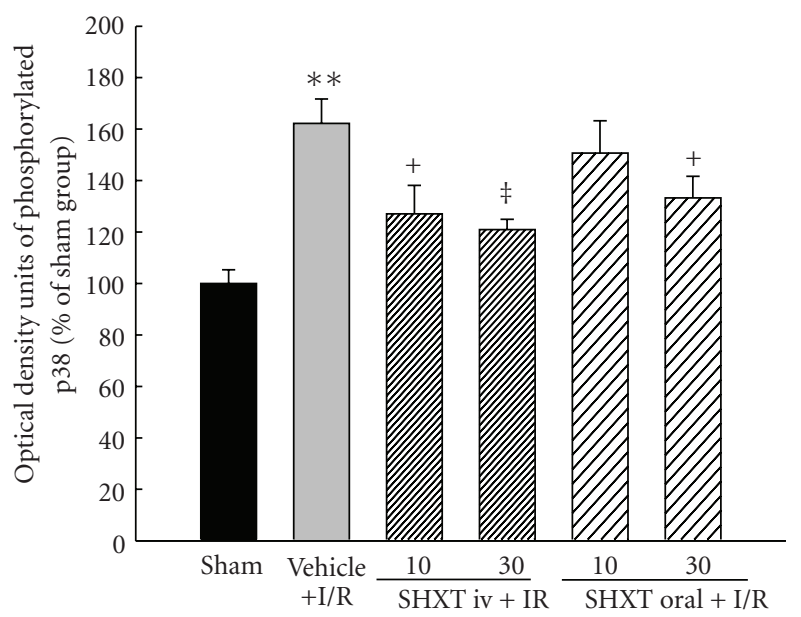

(b)
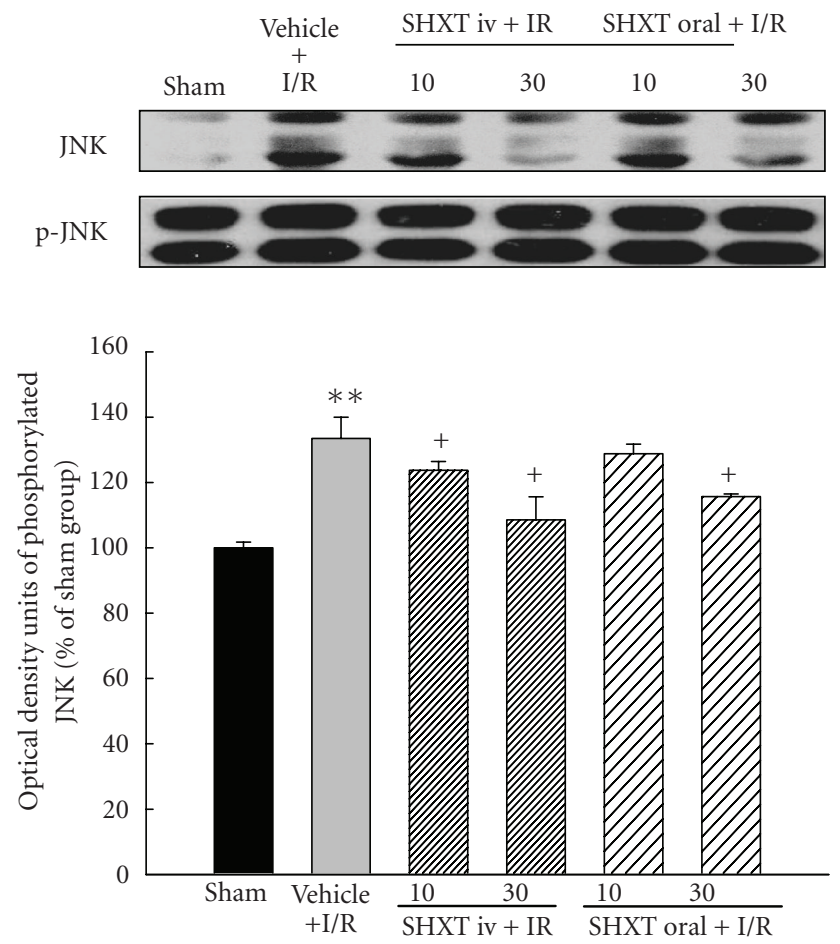

(c)

FIGURE 4: Effects of SHXT on activations of ERK1/2 (a), p38 (b) and JNK (c) induced by I/R. Each value represents the mean \pm SEM of six rats. ${ }^{* *} P<.01$ versus sham group; ${ }^{+} P<.05,{ }^{\ddagger} P<.01$ versus vehicle-treated rats. ANOVA followed by Dunnett's test. 


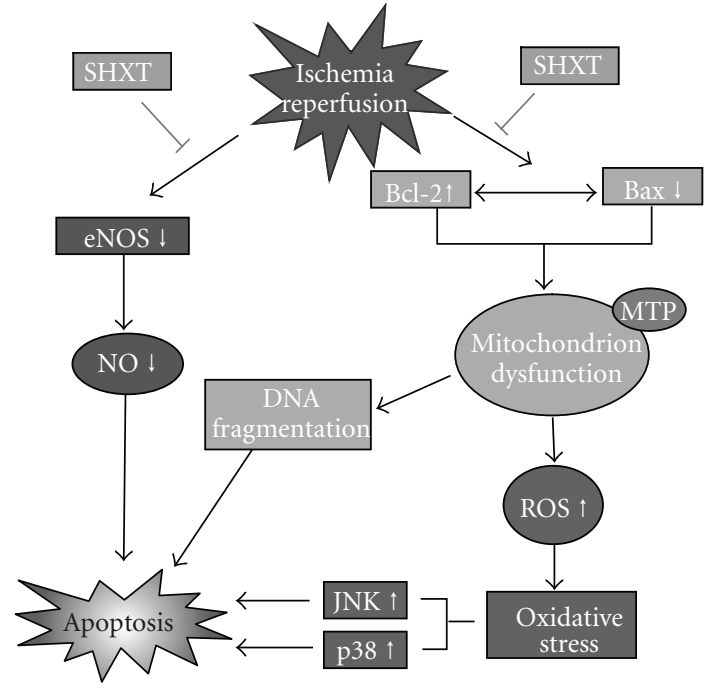

SHXT = San huang-Xie-Xin-tang

$\longrightarrow$ Inhibition

Figure 5: Proposed mechanisms of the cardioprotective effects of SHXT on ischemia/reperfusion-induced myocardial apoptosis according to results in the present study. SHXT can significantly inhibit myocardial apoptosis induced by ischemia/reperfusion, at least in part, by activating eNOS expression and blunting MAPKmediated cascades.

In conclusion, these results demonstrate for the first time that SHXT, a Chinese herbal medicine, has potent cardioprotective effects with anti-apoptosis in rats with myocardial I/R injury, and that these antiapoptotic effects are mediated partly by eNOS and MAPK pathways (Figure 5). This study implicates that SHXT is a potentially useful drug that can be applied clinically in the prevention or treatment of myocardial injury caused by I/R.

\section{Acknowledgments}

The authors would like to thank Ms Li-Ying Chen for technical assistance.

\section{Funding}

Committee on Chinese Medicine and Pharmacy, Department of Health, Taiwan grant CCMP96-RD-014 to J.-L. Y.

\section{References}

[1] P. R. Hansen, "Myocardial reperfusion injury: experimental evidence and clinical relevance," European Heart Journal, vol. 16, no. 6, pp. 734-740, 1995.

[2] M. Akhlaghi and B. Bandy, "Mechanisms of flavonoid protection against myocardial ischemia-reperfusion injury," Journal of Molecular and Cellular Cardiology, vol. 46, no. 3, pp. 309317, 2009.
[3] D. S. Burley and G. F. Baxter, "Pharmacological targets revealed by myocardial postconditioning," Current Opinion in Pharmacology, vol. 9, no. 2, pp. 177-188, 2009.

[4] Y. T. Shih, D. C. Wu, C. M. Liu, Y. C. Yang, I. J. Chen, and Y. C. Lo, "San-Huang-Xie-Xin-Tang inhibits Helicobacter pylori-induced inflammation in human gastric epithelial AGS cells," Journal of Ethnopharmacology, vol. 112, pp. 537-544, 2007.

[5] Y. C. Lo, Y. L. Lin, K. L. Yu et al., "San-Huang-Xie-Xin-Tang attenuates inflammatory responses in lipopolysaccharideexposed rat lungs," Journal of Ethnopharmacology, vol. 101, no. 1-3, pp. 68-74, 2005.

[6] Y.-C. Lo, P.-L. Tsai, Y.-B. Huang et al., "San-Huang-Xie-XinTang reduces lipopolysaccharides-induced hypotension and inflammatory mediators," Journal of Ethnopharmacology, vol. 96, no. 1-2, pp. 99-106, 2005.

[7] H. C. Chen and M. T. Hsieh, "Hemodynamic effects of "sanhuang-hsieh-hsin-tang" in patients with essential hypertension," American Journal of Chinese Medicine, vol. 14, no. 3-4, pp. 153-156, 1986.

[8] H. C. Chen and M. T. Hsieh, "Two-year experience with "San-Huang-Hsieh-Hsin-Tang" in essential hypertension," The American Journal of Chinese Medicine, vol. 14, no. 1-2, pp. 51-58, 1986.

[9] Y. T. Shih, I. J. Chen, Y. C. Wu, and Y. C. Lo, "San-Huang-XieXin-Tang protects against activated microglia- and 6-OHDAinduced toxicity in neuronal SH-SY5Y cells," Evidence-Based Complementary and Alternative Medicine.

[10] Y.-B. Huang, P.-C. Wu, C.-S. Su, Y.-C. Wu, and Y.-H. Tsai, "Simultaneous quantification of twelve bioactive components in San-Huang-Xie-Xin-Tang by HPLC," Phytochemical Analysis, vol. 17, no. 6, pp. 439-446, 2006.

[11] C. S. Shia, Y. C. Hou, S. H. Juang, S. Y. Tsai, P. H. Hsieh, L. C. Ho et al., "Metabolism and pharmacokinetics of SanHuang-Xie-Xin-Tang, a polyphenol-rich Chinese medicine formula, in rats and ex-vivo antioxidant activity," EvidenceBased Complementary and Alternative Medicine.

[12] J. A. Marin-Neto, B. C. Maciel, A. L. Secches, and L. Gallo Jr., "Cardiovascular effects of berberine in patients with severe congestive heart failure," Clinical Cardiology, vol. 11, no. 4, pp. 253-260, 1988.

[13] X.-H. Zeng, X.-J. Zeng, and Y.-Y. Li, "Efficacy and safety of berberine for congestive heart failure secondary to ischemic or idiopathic dilated cardiomyopathy," American Journal of Cardiology, vol. 92, no. 2, pp. 173-176, 2003.

[14] L. Zheng, Z. Zhou, D. Tao, and T. Lan, "Protective effect of berberine on cardiac myocyte injured by ischemiareperfusion," Sichuan Da Xue Xue Bao Yi Xue Ban, vol. 34, no. 3, pp. 452-454, 2003 (Chinese).

[15] A. Y. H. Woo, C. H. K. Cheng, and M. M. Y. Waye, "Baicalein protects rat cardiomyocytes from hypoxia/reoxygenation damage via a prooxidant mechanism," Cardiovascular Research, vol. 65, no. 1, pp. 244-253, 2005.

[16] W.-T. Chang, Z.-H. Shao, J.-J. Yin et al., "Comparative effects of flavonoids on oxidant scavenging and ischemia-reperfusion injury in cardiomyocytes," European Journal of Pharmacology, vol. 566, no. 1-3, pp. 58-66, 2007.

[17] D. Y. Rhyu, K. S. Kang, M. Sekiya, and T. Yokozawa, "Antioxidant effect of Wen-Pi-Tang and its component crude drugs on oxidative stress," American Journal of Chinese Medicine, vol. 35, no. 1, pp. 127-137, 2007. 
[18] J.-C. Liang, H.-R. Chen, C.-C. Chiu, S.-F. Liou, I.-J. Chen, and J.-L. Yeh, "Protective effect of labedipinedilol-A, a novel dihydropyridine-type calcium channel blocker, on myocardial apoptosis in ischemia-reperfusion injury," Life Sciences, vol. 79, no. 13, pp. 1248-1256, 2006.

[19] M. J. A. Walker, M. J. Curtis, D. J. Hearse et al., "The Lambeth Conventions: guidelines for the study of arrhythmias in ischaemia, infarction, and reperfusion," Cardiovascular Research, vol. 22, no. 7, pp. 447-455, 1988.

[20] A. T. Demiryürek, G. Yildiz, S. Eşiyok, and S. Altuğ, "Protective effects of poly (ADP-ribose) synthase inhibitors on digoxininduced cardiotoxicity in guinea-pig isolated hearts," Pharmacological Research, vol. 45, no. 3, pp. 189-194, 2002.

[21] J. Fu, G. Lin, Z. Wu et al., "Anti-apoptotic role for C1 inhibitor in ischemia/reperfusion-induced myocardial cell injury," Biochemical and Biophysical Research Communications, vol. 349, no. 2, pp. 504-512, 2006.

[22] P. Mukhopadhyay, S. Bátkai, M. Rajesh et al., "Pharmacological inhibition of CB1 cannabinoid receptor protects against doxorubicin-induced cardiotoxicity," Journal of the American College of Cardiology, vol. 50, no. 6, pp. 528-536, 2007.

[23] D. J. Waxman and P. S. Schwartz, "Harnessing apoptosis for improved anticancer gene therapy," Cancer Research, vol. 63, no. 24, pp. 8563-8572, 2003.

[24] R. Moudgil, V. Menon, Y. Xu, S. Musat-Marcu, D. Kumar, and B. I. Jugdutt, "Postischemic apoptosis and functional recovery after angiotensin II type 1 receptor blockade in isolated working rat hearts," Journal of Hypertension, vol. 19, no. 6, pp. 1121-1129, 2001.

[25] Z. Chen, C. C. Chua, Y.-S. Ho, R. C. Hamdy, and B. H. L. Chua, "Overexpression of $\mathrm{Bcl}-2$ attenuates apoptosis and protects against myocardial I/R injury in transgenic mice," American Journal of Physiology, vol. 280, no. 5, pp. H2313-H2320, 2001.

[26] G. Yang, Z. Fang, Y. Liu, H. Zhang, X. Shi, Q. Ji et al., "Protective effects of Chinese traditional medicine Buyang Huanwu decoction on myocardial injury," Evidence-Based Complementary and Alternative Medicine.

[27] H. M. Razavi, J. A. Hamilton, and Q. Feng, "Modulation of apoptosis by nitric oxide: implications in myocardial ischemia and heart failure," Pharmacology and Therapeutics, vol. 106, no. 2, pp. 147-162, 2005.

[28] S. P. Jones, W. G. Girod, A. J. Palazzo et al., "Myocardial ischemia-reperfusion injury is exacerbated in absence of endothelial cell nitric oxide synthase," American Journal of Physiology, vol. 276, no. 5, pp. H1567-H1573, 1999.

[29] M. S. Sumeray, D. D. Rees, and D. M. Yellon, "Infarct size and nitric oxide synthase in murine myocardium," Journal of Molecular and Cellular Cardiology, vol. 32, no. 1, pp. 35-42, 2000.

[30] S. Wolfrum, A. Dendorfer, M. Schutt et al., "Simvastatin acutely reduces myocardial reperfusion injury in vivo by activating the phosphatidylinositide 3-kinase/Akt pathway," Journal of Cardiovascular Pharmacology, vol. 44, no. 3, pp. 348-355, 2004.

[31] Y. N. Liu, Z. M. Zhou, and P. Chen, "Evidence that hydroxysafflor yellow A protects the heart against ischaemia-reperfusion injury by inhibiting mitochondrial permeability transition pore opening," Clinical and Experimental Pharmacology and Physiology, vol. 35, pp. 211-216, 2008.

[32] C.-C. Hsieh and J. Papaconstantinou, "Thioredoxin-ASK1 complex levels regulate ROS-mediated p38 MAPK pathway activity in livers of aged and long-lived Snell dwarf mice," FASEB Journal, vol. 20, no. 2, pp. 259-268, 2006.
[33] K. Shimada, M. Nakamura, E. Ishida, M. Kishi, and N. Konishi, "Roles of p38- and c-jun NH2-terminal kinase-mediated pathways in 2-methoxyestradiol-induced p53 induction and apoptosis," Carcinogenesis, vol. 24, no. 6, pp. 1067-1075, 2003.

[34] T.-L. Yue, C. Wang, J.-L. Gu et al., "Inhibition of extracellular signal-regulated kinase enhances ischemia/reoxygenationinduced apoptosis in cultured cardiac myocytes and exaggerates reperfusion injury in isolated perfused heart," Circulation Research, vol. 86, no. 6, pp. 692-699, 2000.

[35] M. Slevin, K. Krupinski, A. Slowik, F. Rubio, A. Szczudlik, and J. Gaffney, "Activation of MAP kinase (ERK-1/ERK-2), tyrosine kinase and VEGF in the human brain following acute ischaemic stroke," NeuroReport, vol. 11, no. 12, pp. 2759-2764, 2000.

[36] S. Zhuang and R. G. Schnellmann, "A death-promoting role for extracellular signal-regulated kinase," Journal of Pharmacology and Experimental Therapeutics, vol. 319, no. 3, pp. 991997, 2006.

[37] J. Liu, W. Mao, B. Ding, and C.-S. Liang, "ERKs/p53 signal transduction pathway is involved in doxorubicin-induced apoptosis in H9c2 cells and cardiomyocytes," American Journal of Physiology, vol. 295, no. 5, pp. H1956-H1965, 2008. 


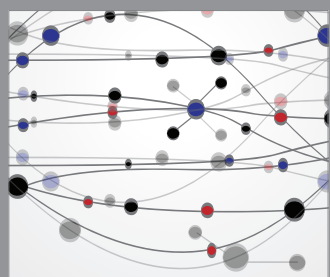

The Scientific World Journal
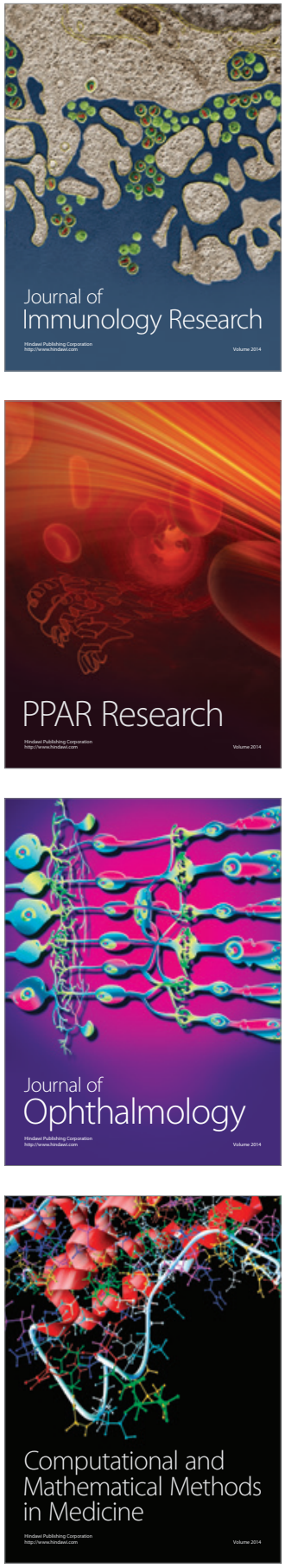

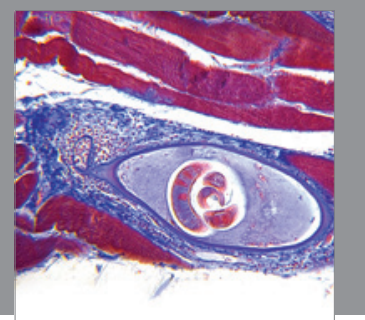

Gastroenterology

Research and Practice
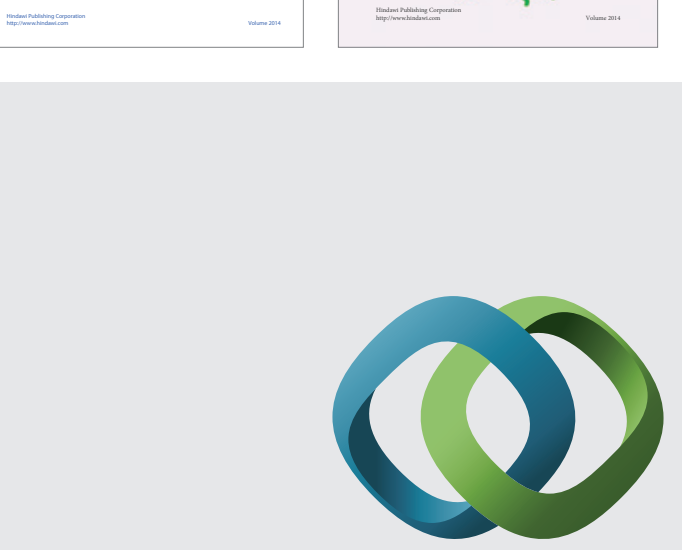

\section{Hindawi}

Submit your manuscripts at

http://www.hindawi.com
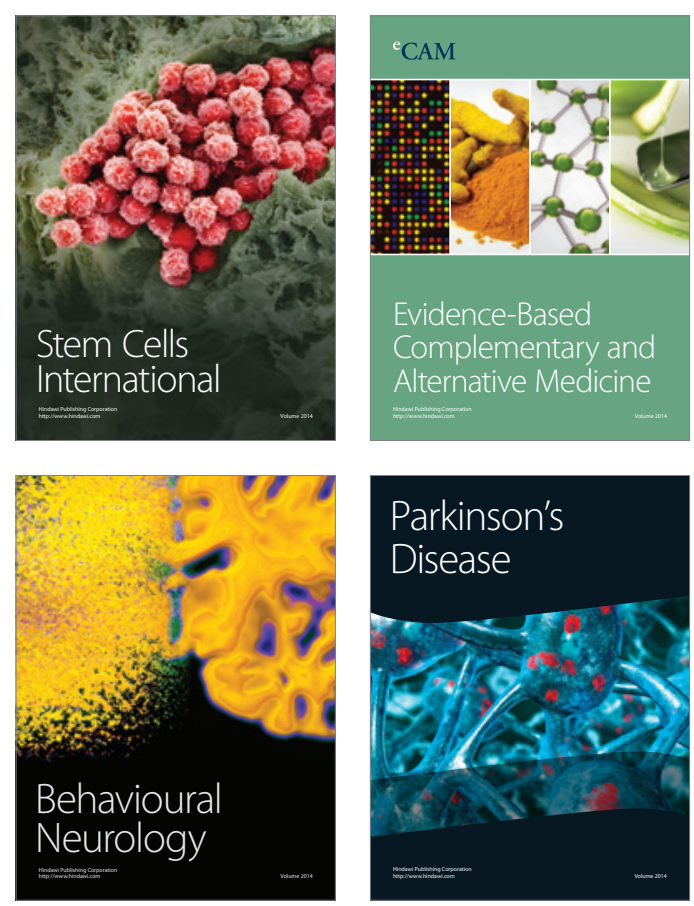

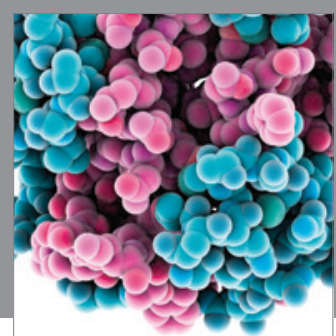

Journal of
Diabetes Research

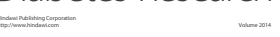

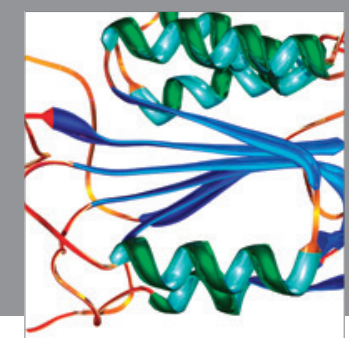

Disease Markers
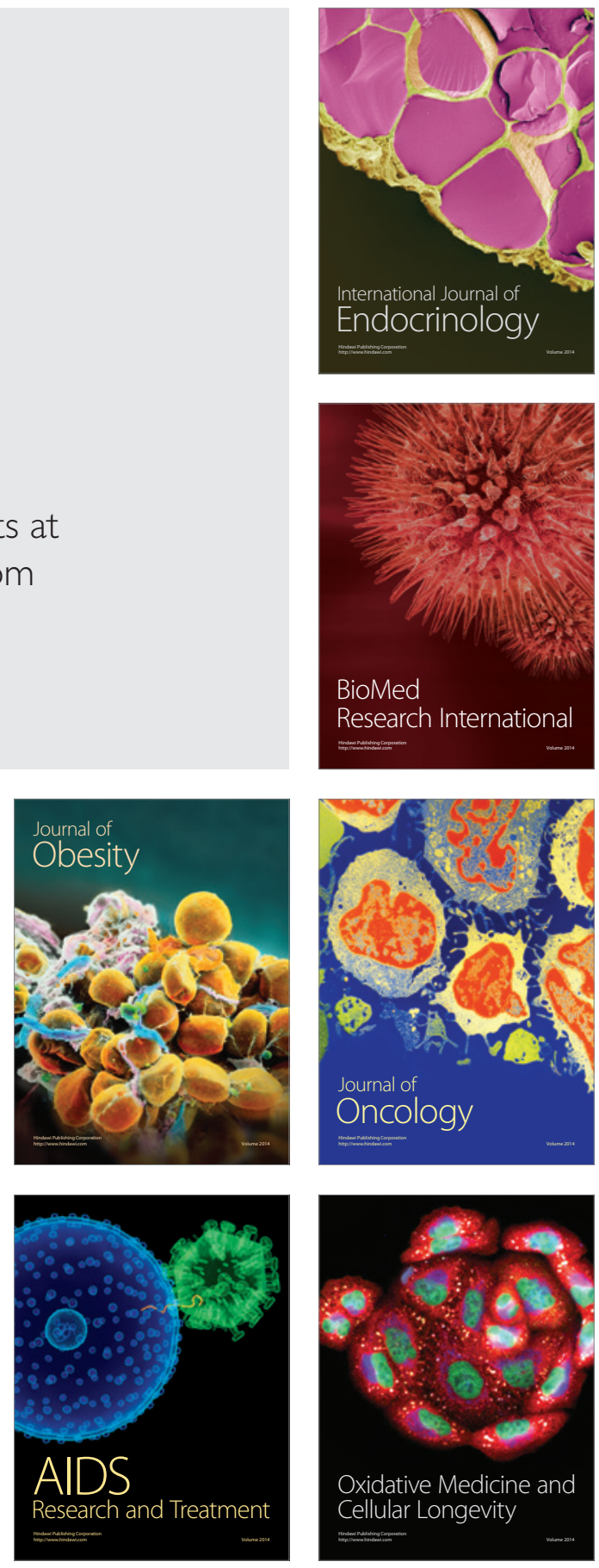